\title{
Identification of copy number variation in French dairy and beef breeds using next-generation sequencing
}

\author{
Rabia Letaief ${ }^{*}$, Emmanuelle Rebours ${ }^{1}$, Cécile Grohs ${ }^{1}$, Cédric Meersseman ${ }^{1,2}$, Sébastien Fritz ${ }^{1,3}$, Lidwine Trouilh" \\ Diane Esquerré ${ }^{5}$, Johanna Barbieri ${ }^{5}$, Christophe Klopp ${ }^{6}$, Romain Philippe ${ }^{2}$, Véronique Blanquet ${ }^{2}$, \\ Didier Boichard ${ }^{1}$, Dominique Rocha ${ }^{1}$ and Mekki Boussaha ${ }^{1}$
}

\begin{abstract}
Background: Copy number variations (CNV) are known to play a major role in genetic variability and disease pathogenesis in several species including cattle. In this study, we report the identification and characterization of CNV in eight French beef and dairy breeds using whole-genome sequence data from 200 animals. Bioinformatics analyses to search for CNV were carried out using four different but complementary tools and we validated a subset of the CNV by both in silico and experimental approaches.

Results: We report the identification and localization of 4178 putative deletion-only, duplication-only and CNV regions, which cover $6 \%$ of the bovine autosomal genome; they were validated by two in silico approaches and/or experimentally validated using array-based comparative genomic hybridization and single nucleotide polymorphism genotyping arrays. The size of these variants ranged from $334 \mathrm{bp}$ to $7.7 \mathrm{Mb}$, with an average size of $\sim 54 \mathrm{~kb}$. Of these 4178 variants, 3940 were deletions, 67 were duplications and 171 corresponded to both deletions and duplications, which were defined as potential CNV regions. Gene content analysis revealed that, among these variants, 1100 deletions and duplications encompassed 1803 known genes, which affect a wide spectrum of molecular functions, and 1095 overlapped with known QTL regions.
\end{abstract}

Conclusions: Our study is a large-scale survey of CNV in eight French dairy and beef breeds. These CNV will be useful to study the link between genetic variability and economically important traits, and to improve our knowledge on the genomic architecture of cattle.

\section{Background}

For the first time in 2004, copy number variations (CNV) were reported as a new form of genomic alteration [1, 2]. CNV are defined as gains or losses of DNA segments ranging from $50 \mathrm{bp}$ to several megabases $(\mathrm{Mb})$. $\mathrm{CNV}$ are considered to be polymorphic genetic markers and are inherited across generations [3]. At the genome level, $\mathrm{CNV}$ are less frequent than single nucleotide polymorphisms (SNPs) and small insertions and deletions (InDel), but they can have a greater functional and evolutionary

\footnotetext{
*Correspondence: rabia.letaief@inra.fr

${ }^{1}$ GABI, INRA, AgroParisTech, Université Paris-Saclay, 78352 Jouy-en-Josas, France

Full list of author information is available at the end of the article
}

impact. For example, by modifying the genome organization, $\mathrm{CNV}$ can affect gene expression and therefore certain phenotypes of interest [4].

$\mathrm{CNV}$ and their impact have been extensively studied in several species, particularly in humans, in which they are known to cause several genetic diseases. For example, a 2-kb deletion located upstream of the IRGM (immunity related GTPase $M$ ) gene is linked with Crohn's disease [5], a CNV located within the TSPAN8 (tetraspanin 8) gene is associated with type 2 diabetes [6], and a duplication within the CCL3L1 (C-C motif chemokine ligand 3 like 1) gene is involved in HIV susceptibility [7].

In domesticated animals, CNV are also linked with several phenotypic traits. For example, two duplications 
that overlap with the KIT (KIT proto-oncogene receptor tyrosine kinase) and ASIP (agouti signaling protein) genes are responsible for white coat color in pigs and sheep, respectively $[8,9]$. In chickens, the pea-comb phenotype is associated with a duplication within the SOX5 (SRYbox 5) gene $[10,11]$. In ridgeback dogs, a 133-kb duplication is located within the genomic region that contains the FGF3 (fibroblast growth factor 3), FGF4 (fibroblast growth factor 4), FGF19 (fibroblast growth factor 19), and ORAOV1 (oral cancer overexpressed 1) genes and causes both hair ridge and a predisposition to dermoid sinus [12]. In cattle, anhidrotic ectodermal dysplasia is induced by a deletion in the ED1 (anhidrotic ectodermal dysplasia) gene [13], and polled and multisystemic syndrome is caused by a deletion that knocks out the ZEB2 (zinc finger E-box binding homeobox 2) gene [14].

Both array-based comparative genomic hybridization (CGH) and SNP arrays have long been widely used for the detection of CNV. However, these two approaches are not very efficient and lack the sensitivity needed to detect a wide range of CNV [15-18]. For example, the resolution of the array-based CGH approach depends on the number, size, and quality of the probes fixed on the array [15-18]. Thus, with a low-density array, it is difficult to detect all the small variants. Similarly, detection of CNV with SNP genotyping arrays depends on the nature of the SNPs included and their distribution within the genome and if the density of the SNP array is low, detection of small variants is unlikely.

The advent of whole-genome sequencing, coupled with major bioinformatics developments, have profoundly modified strategies used to detect CNV. Unlike arraybased CGH and SNP array platforms, the next-generation sequencing (NGS)-based approach can identify a wide range of $\mathrm{CNV}$, ranging in size from tens of nucleotides to several $\mathrm{Mb}$, with accurate localization of breakpoints.

In this study, we performed a genome-wide characterization of $\mathrm{CNV}$ in cattle using four software packages based on three different approaches. We performed a bioinformatics search for $\mathrm{CNV}$ by exploring wholegenome sequencing data from 200 animals that represented eight French dairy and beef breeds.

\section{Methods}

\section{Animal ethics}

Most whole-genome sequences used in this study were already available in our laboratory (see [19] for more details), and thus no animal experimentation was necessary for this study. A small part of our dataset was generated from 23 genomic DNA samples that were obtained from muscle tissue collected at commercial slaughterhouses. Five other genomic DNA samples were prepared from sperm collected from semen straws that were provided by approved commercial artificial insemination stations as part of their regular semen collection process.

\section{Genomic DNA extraction and whole-genome sequencing} Details on the extraction of genomic DNA for 172 of the animals are in [19] and DNA extraction for the remaining 28 animals was performed using the Wizard Genomic DNA Purification kit (Promega, Charbonnières-les-Bains, France). Each purified DNA sample was quality-controlled by agarose gel electrophoresis. DNA concentration was then measured with a Nanodrop ND-100 instrument (Thermo Fisher Scientific, Ilkirch, France). Genomic DNA library construction and sequencing for the 200 animals were performed as previously described [20]. All sequences were then aligned to the UMD3.1 reference genome sequence with the Burrows-Wheeler aligner (BWA) [21].

\section{DNA sampling}

Two hundred French cattle were selected for sequencing as representative of four main dairy, i.e. Brown Swiss, Holstein, Montbéliarde, and Normande and four main beef breeds, i.e. Blonde d'Aquitaine, Charolaise, Limousine, and Rouge des Prés (Table 1) and Table S1 (see Additional file 1: Table S1). Of these 200 animals, 14 were sequenced at least twice. In addition, two sire-dam-son trios (both Montbéliarde) and 16 sire-son pairs (six Normande, eight Montbéliarde, and two Holstein pairs) were included.

\section{Detection of CNV}

Computational approaches for searching CNV in wholegenome sequence data involved four commonly used tools. CNVnator v0.3 [22] identified CNV using a readdepth (RD) approach within genomic windows of $250 \mathrm{bp}$. BreakDancer v1.3.6 [23] was run with default parameters to detect $\mathrm{CNV}$ with the paired-end mapping (PEM) approach. Both Pindel v2.5 [24] and DELLY v0.6.1 [25] software packages use a PEM-based strategy followed by a split-read (SR)-based approach to determine the type and the size of the predicted variant. Pindel and DELLY were used with default parameters.

\section{Analysis of CNV}

For each animal, first we excluded all variants for which the breakpoint positions were located within a 100-bp window that contained a gap in the reference sequence. Then, we filtered out all variants for which more than $25 \%$ of the bases consisted of gaps. Information about the location of all unknown sequences within the UMD3.1 reference genome sequence was downloaded from the NCBI database (ftp://ftp.ncbi.nlm.nih.gov/genomes/ Bos_taurus/Assembled_chromosomes/agp/). Finally, we 
Table 1 Distribution of animals per breed and sequencing coverage

\begin{tabular}{lcclll}
\hline Breed & Number of animals & Coverage min-max & Coverage mean & Percentage of chimeric reads & Breed type \\
\hline Blonde d'Aquitaine & 25 & $11-26$ & 15 & $1.1 \pm 0.1$ & Beef \\
Brown Swiss & 3 & $9-12$ & 10 & $0.3 \pm 0.03$ & Dairy \\
Charolaise & 25 & $11-25$ & 15 & $1.3 \pm 0.2$ & Beef \\
Holstein & 56 & $8-21$ & 13 & $0.8 \pm 0.9$ & Dairy \\
Limousine & 34 & $8-25$ & 14 & $1.6 \pm 0.8$ & Beef \\
Montbéliarde & 31 & $9-28$ & 15 & $1.6 \pm 1.1$ & Dairy \\
Normande & 23 & $8-33$ & 12 & $2.3 \pm 0.9$ & Dairy \\
Rouge des Prés & 3 & $16-31$ & 21 & $1.3 \pm 0.1$ & Beef \\
Total & 200 & $8-33$ & 14.4 & $1.4 \pm 1.5$ & \\
\hline
\end{tabular}

selected all variants that were supported by a minimum of three reads and retained only those that were predicted by at least two different tools. In addition, a variant that was predicted by both Pindel and DELLY was retained only if these two methods identified the corresponding breakpoint positions that were within $100 \mathrm{bp}$ of each other (i.e. the $5^{\prime}$ breakpoint indicated by Pindel was within $100 \mathrm{bp}$ of the breakpoint identified by DELLY, and the same for the $3^{\prime}$ breakpoint). For all other combinations of tools, we applied a 90\% reciprocal overlap (RO) threshold for defining $\mathrm{CNV}$ as belonging to the same region; otherwise, they were considered as distinct regions (Fig. 1).

Variants that passed these filtering criteria were subsequently checked in other samples. A given CNV was defined as common to at least two samples when the predicted region in one sample had at least $70 \%$ reciprocal overlap with the CNV region predicted in another sample. The resulting overlapping variants were then used to define potential CNV regions (CNVR). The new breakpoint positions and the size of these CNVR were defined as follows: (1) the $5^{\prime}$ and $3^{\prime}$ genomic positions of the CNVR corresponded respectively to the lowest $5^{\prime}$ and the highest $3^{\prime}$ positions of all overlapping variants identified in the previous steps; and (2) the size of the CNVR was defined as the interval between the new $5^{\prime}$ and $3^{\prime}$ breakpoint positions.

\section{Validation of CNV Mendelian approach}

CNV were validated by two in silico and two experimental approaches. First, two family trios and 16 sireson pairs from our dataset were sequenced. Since CNV should be inherited from parent to son, we reported only $\mathrm{CNV}$ that were present in either of the parents and the offspring in trios or in both sire and son in sire-son pairs.

\section{Twice-sequenced approach}

Second, we used 14 animals for which we had sequencing data generated from at least two different sequencing runs in order to estimate the number of shared predicted CNV between the two datasets. Theoretically, for a given animal, the same CNV should be present in each of the two independent sequences.

\section{Array-based CGH approach}

The third validation approach involved array-based comparative genomic hybridization $(\mathrm{CGH})$ analysis. In this study, $\mathrm{CGH}$ experiments were performed using the Agilent CGH array (SurePrint G3 Bovine CGH Microarray, $4 \times 180 \mathrm{~K}$ ), which contained 152,934 oligonucleotide probes, each 45 to 60 nucleotides long. Adjacent probes were on average 16,376 bp apart on the UMD3.1 reference genome. DNA for 17 animals was analyzed using this CGH microarray, including DNA from Dominette (the animal that was used to generate the bovine reference genome), which was used for normalization steps. The 17 animals were also sequenced in this study (the detailed protocol for array-based CGH is available in Additional file 2). Briefly, $600 \mathrm{ng}$ of fragmented DNA was labeled with Cy3- or Cy5-labeled nucleotides (tested DNA and control DNA, respectively). Tested and control DNA were then co-hybridized in the Agilent system and the array was scanned on the MS200 scanner (TECAN) according to the manufacturer's instructions and as performed in other studies [26, 27]. Fluorescence intensities were normalized and quality was checked using the Feature Extraction software v11.5.1.1 from Agilent. Then, the aberration detection method 2 (ADM-2) algorithm was applied using Agilent Genomic Workbench software (v7.0.4.0) to detect variants. Potential variants were detected by analyzing aberrations in the normalized fluorescence intensities 


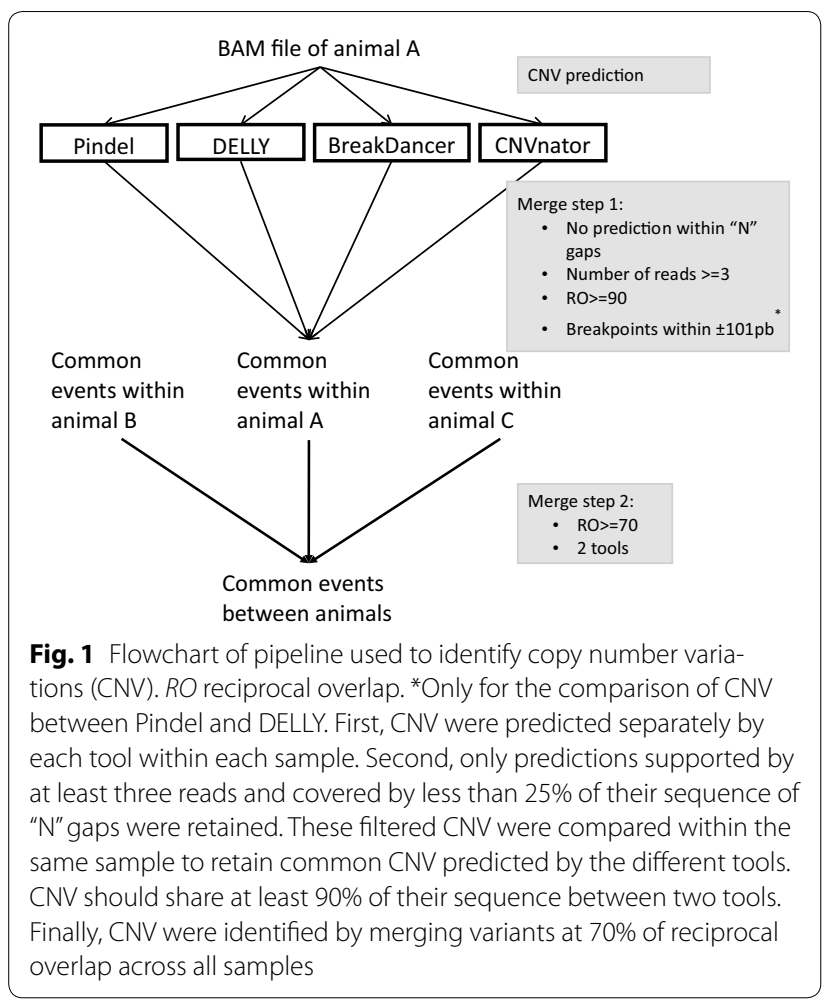

relative to the reference DNA sample, Dominette (log 2 ratio). A CNV was retained if at least three consecutive probes supported it. Finally, CNVR were defined by comparing identified CNV across samples. Two CNV belonged to the same CNVR if they shared at least 50\% of their sequence.

\section{Custom SNP genotyping approach}

Finally, we selected 122 deletion-only, duplication-only, or $\mathrm{CNV}$ regions for testing in genotyping assays using the Illumina bovine low-density BeadChip (Infinium ${ }^{\circledR}$ BovineLD v6.0: LDv6) [28]. In this chip, each variant was represented by at least three SNPs that were uniformly spaced. These SNPs were chosen from the SNP catalog published by Boussaha et al. [19]. Overall, 1008 SNPs were genotyped for 14,082 animals from 18 different breeds by LABOGENA SA (France), following the manufacturer's recommendations. Total signal intensity (Log R Ratio: LRR) and allelic intensity ratio (B allele frequency: BAF) of the SNPs for each sample were collected and then analyzed with PennCNV (2011Jun16 version) [29] to identify CNV. Only samples with an LRR standard deviation less than 0.3 and a BAF drift less than 0.01 were considered for detection. Then, the resulting variants were compared to those predicted with WGS data. As for the array-based CGH approach, two variants were considered as identical if at least $50 \%$ of their sequence were shared and if they passed all other in silico approaches.

\section{Statistics}

All reported statistics were calculated using $\mathrm{R}$ software. The Pearson method was used first to assess the correlation between reported variants and coverage rate, and second to calculate the correlation between the number of variants and chromosome size. The Chi square test was used to evaluate within-breed genetic variability. This within-breed genetic variability was assessed by comparing the number of predicted variants to the expected number, which was calculated as the ratio between number of predicted variants and number of sequenced animals within each breed.

\section{Comparison of CNVR with known bovine CNV}

We compared our $\mathrm{CNV}$ dataset with those previously published for cattle [4, 20, 30-34] and with the publicly available Genomic Variants archive (DGVa) database of EMBL-EBI (http://www.ebi.ac.uk/dgva). These publicly available $\mathrm{CNV}$ were detected using array-based CGH [30], SNP genotyping arrays [31, 32], and whole-genome sequencing $[4,20,33,34]$. The comparison was carried out using the Bedtools software package [35]. Given the differences between platforms, definitions of CNV, and methods of CNVR construction used, two CNV were considered as shared when there was at least $50 \%$ of reciprocal overlap between the two regions.

\section{Gene content and gene ontology}

First, functional elements that were located within or overlapping with deletion-only, duplication-only, and $\mathrm{CNV}$ regions were identified by using a custom python script (available upon request) that was coupled with the "intersectBed" option of the Bedtools package [35]. Gene content was analyzed by using the bovine Ensembl genebuild database (version 89) retrieved from the BioMart database (http://www.ensembl.org/biomart/). In total, 24,616 bovine genes were downloaded. For each gene that was located within a CNVR or overlapping with a CNVR, we used Ensembl information to verify whether there were known paralogous genes.

Second, we used the public animal QTLdb database, release 32 [36], to check whether the variants included in our panel were located within or overlapped with publicly available bovine quantitative trait loci (QTL).

Finally, gene ontology (GO) and Kyoto Encyclopedia of Genes and Genomes (KEGG) pathway analyses were performed using the PANTHER classification system v11.1 [37]. After Bonferroni correction, enriched GO terms within biological processes, cellular components, and molecular functions were identified. 


\section{Results and discussion}

\section{Sequencing data}

One hundred seventy-two French beef and dairy animals were sequenced as described by Boussaha et al. [19] (see Additional file 1: Table S1). Sequencing details for the 28 whole-genome sequences that were obtained for this study are in Table S1 (Additional file 1: Table S1). As in Hoze et al. [38], 180 sires of the 200 sequenced animals were chosen based on their marginal contribution to their population based on pedigree information, as defined by Boichard et al. [39], and computed using the PEDIG software [40]. Eighteen of the remaining 20 animals were sons of some of these 180 selected sires. In addition, two mothers of some of the 18 sons were chosen.

Paired-end sequencing produced 71.51 billion pairedends read, of which 69.46 billion (96.5\%) were correctly mapped to the UMD3.1 reference genome sequence. The average insert size was $321 \mathrm{bp}$ and the average wholegenome sequencing coverage was $14.4 \mathrm{x}$, ranging from $8 \times$ to $33 \times$ (see Additional file 1: Table S1).

\section{Deletion, duplication, and CNV calls}

Deletions, duplications, and CNV regions were predicted using four tools. To define potential CNVR, raw events predicted by all four tools were merged and further analyzed (Fig. 1). Overall, we detected 19,077 deletion and duplication events predicted by at least two tools (see Additional file 3: Table S2).

\section{Validation of CNVR}

Given the huge number of detected variants and the likelihood of a relatively high rate of false positives due to the number of approaches used, we decided to apply multiple criteria to retain the most reliable variants. Thus, all detected variants were validated by applying four strategies, in silico and experimentally, and only the validated ones were considered for further analyses.

\section{Mendelian approach}

Analysis of trios and parent-offspring pairs revealed that $57 \%$ (4596 of 8088 ) of the identified variants were present in at least one parent and one of its offspring (Fig. 2), Table S1 (see Additional file 1: Table S1) and Table S3 (see Additional file 4: Table S3). Of these 4596 variants, almost 33\% were present in all three members of the trio (sire, dam, and offspring) and in all offspring of a given sire (in cases in which a sire had several offspring). The percentage of variants that were transmitted from either of the parents to the offspring was highly variable, ranging from 12 to $72 \%$, and was highly correlated with sequencing depth (Pearson correlation score $\rho=0.75, p$ value $=1.6 \mathrm{e}-4)($ see Additional file 5: Table S4).

\section{Twice-sequenced approach}

Next, we compared the variants detected in wholegenome sequence data generated from two or more sequencing runs of the same individual. In total, 7394 of the 9266 variants that were predicted for these 14 animals were found in both sets of sequence data by at least one CNV detection tool (see Additional file 6: Table S5). The overall concordance rate was around $80 \%$. Of these 7394 variants, 44\% (3261) were validated in at least two different breeds, $38 \%$ were validated only in Montbéliarde, $4 \%$ only in Charolaise, $6 \%$ only in Limousine, $4 \%$ only in Rouge des Prés, and $2 \%$ only in Blonde d'Aquitaine. Moreover, around 56\% (4163) of these variants were also confirmed by the Mendelian strategy.

\section{Array-based CGH approach}

Array-based $\mathrm{CGH}$ analysis of whole-genome sequence data from 17 individuals resulted in the identification of 68 variants (see Additional file 7: Table S6). The detection of a given type of variants for one animal with the array-based $\mathrm{CGH}$ method was compared to that with the whole-genome sequence method (reciprocal overlap $(\mathrm{RO}) \geq 50 \%$ ); of these 68 variants, $34 \%$ (23 variants) were found by both approaches. In addition, when variants detected by array-based CGH were compared with raw variants data that were predicted using wholegenome sequence data prior to merging, we retrieved $18 \%$ (12 variants) more than in the analysis that used only merged variants. These 12 variants were mostly predicted with CNVnator (92\%, 11 variants) (see Additional file 7: Table S6). Following this comparison, 22\% (15 variants) of the remaining variants detected by array-based CGH were found in the whole-genome sequence dataset if we relaxed the $\mathrm{RO}$ threshold to $1 \%$; the fact that these variants were excluded from the sequence approach showed the stringency of the criteria that we used to define variants (see Additional file 7: Table S6). The remaining 26\% (18 variants) of the variants detected by array-based $\mathrm{CGH}$ not found with whole-genome sequence data were located in poorly sequenced regions. Among the 23 variants detected by both array-based $\mathrm{CGH}$ and wholegenome sequence approaches, eight were also found in animals sequenced in at least two sequencing runs, and three were also validated by the Mendelian approach.

\section{Custom SNP genotyping approach}

Finally, we used SNP genotyping assays to test 122 variants, which were found by the Mendelian and twicesequenced approaches. These variants were selected based on their frequency $(\geq 10 \%)$ in at least one of the three main French dairy breeds (Montbéliarde, Normande and Holstein). For each variant, we selected at 


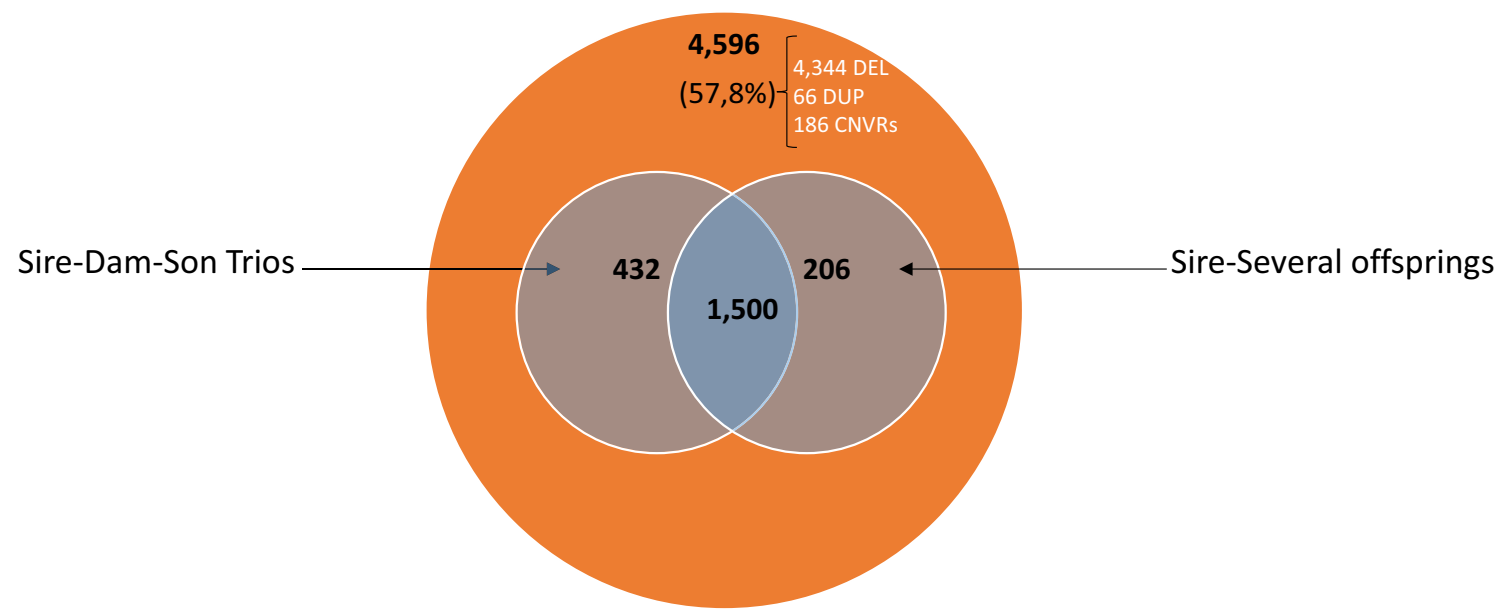

Fig. 2 Number of variants supported by Mendelian inheritance. The total number of variants supported by Mendelian inheritance is indicated in the orange circle (two family trios and 16 sire-son pairs). The sire-dam-son trios legend represents the number of variants found in the offspring and in its parents. Sire-several offspring indicates the number of variants that were predicted for the sire and several of its offspring

least three SNPs from a publicly available SNP dataset [19]. We validated 69 variants (56\%) (see Additional file 8: Table S7). Eight additional variants were retained by applying a RO threshold of $20 \%$. In total, 45 variants that were predicted from WGS data were not captured with the approach based on SNP genotyping data. These variants may have been excluded either during the filtering steps of the SNP genotyping quality control or during the $\mathrm{CNV}$ identification process, in which three SNPs were needed to retain the $\mathrm{CNV}$.

Overall, we retained 4178 variants that were detected by both in silico approaches (Mendelian and twicesequenced approaches) and/or CGH. Of these 4178 variants, $83 \%$ (3464) were predicted by a minimum of three tools and $22.2 \%$ (927) were predicted by all four tools (see Additional file 9: Table S8). Most validated variants were predicted by both DELLY and BreakDancer (4075 variants, 97.5\%). The smallest number of validated variants was identified by the combination of Pindel + CNVnator (1047 variants, 25\%) (Table 2).

Analysis of the distribution of the percentage of validated variants per combination of tools shows that the Pindel + CNVnator combination yielded the highest percentage (1047 validated variants out of 2048 total predicted variants, $51.1 \%$ ) followed by the DELLY + CNVnator combination $(44.2 \%$ i.e. 2139 validated variants out of 4840 total predicted variants). DELLY combined with BreakDancer predicted the largest number of variants but only $23.3 \%$ of these (4075 validated variants out of 17,479 total predicted variants) were validated, which indicates that these tools have a high rate of false positives. This is consistent with previous reports that showed that DELLY outperforms the other tools in terms of discovery, but has a high rate of false positives $[41,42]$. In contrast, all combinations of tools that included Pindel had a higher rate of validated variants (42\%), which reflects the high degree of accuracy of this tool for predicting variants, which is likely due to Pindel using the split-read approach on oneend anchor reads to identify, with high resolution, the breakpoint positions of a variant.

\section{Distribution of variants}

We retained 4178 variants that were validated by both in silico approaches and/or array-based CGH and, across all animals, they represented 6\% $(150 \mathrm{Mb})$ of the UMD3.1 cattle genome assembly. These variants comprised 3940 deletion-only regions, 67 duplication-only regions and 171 CNVR (Table 3).

Analysis of the distribution of variants on the autosomes revealed a significant correlation between the number of predicted variants and chromosome size (Pearson correlation score $\rho=0.91, p$ value $=5.56 \mathrm{e}-12$, Fig. 3a). Bos taurus chromosome 1 (BTA1), 6, and 5 carried the largest number of variants $(277,232$, and 231 , respectively; (see Additional file 10: Table S9), whereas BTA25 had the smallest number (38). However, the correlations between number of predicted variants and proportion of each chromosome covered by variants were quite different (Fig. $3 \mathrm{~b}$ ). The highest percentage of sequence covered by $\mathrm{CNV}(30.2 \%)$ was found for BTA27, which was also the chromosome that was most covered by duplication-only regions $(2.7 \mathrm{Mb}$, CNVR_11771). On the contrary, BTA3, 13 and 27 were the chromosomes that were most enriched in deletiononly regions (15.2, 11.2 and $10.5 \mathrm{Mb}$, respectively). BTA3 and 13 carried the largest deletions that we detected 
Table 2 Percentage of validated variants per combination of tools

\begin{tabular}{lcll}
\hline Combination of tools & $\begin{array}{l}\text { Number } \\
\text { of variants } \\
\text { before vali- } \\
\text { dation }\end{array}$ & $\begin{array}{l}\text { Number } \\
\text { of variants } \\
\text { after valida- } \\
\text { tion }\end{array}$ & $\begin{array}{l}\text { Validation } \\
\text { rate (\%) }\end{array}$ \\
\hline Pindel + DELLY & 7510 & 3169 & 42.2 \\
Pindel + CNVnator & 2048 & 1047 & 51.1 \\
Pindel + BreakDancer & 7364 & 3161 & 42.9 \\
DELLY + CNVnator & 4840 & 2139 & 44.2 \\
DELLY + BreakDancer & 17,479 & 4075 & 23.3 \\
CNVnator + BreakDancer & 6060 & 2327 & 38.4 \\
Total & 19,077 & 4178 & \\
\hline
\end{tabular}

in our dataset i.e. 7.7 $\mathrm{Mb}$ (CNVR_13246) and 6.9 Mb (CNVR_3635), respectively. In addition, two large deletions (CNVR_11676 and CNVR_11898) that together covered $8.6 \mathrm{Mb}$ were identified on BTA27. BTA12 and 23 carried the largest CNVR i.e. 6.3 Mb (CNVR_3252 and CNVR_3296) and 4.3 Mb (CNVR_10026), respectively.

In contrast, the lowest percentage of sequence covered by variants $(0.6 \%)$ was found for BTA2 25 and for BTA 1 (1.1\%) although this chromosome had the largest number of CNV (see Additional file 10: Table S9). The total length of CNV per chromosome was not correlated with chromosome length (Pearson correlation score $\rho=0.12$, $p$ value $=0.54)$.

On average, we identified 1132 variants per individual and this number ranged from 46 to 2957 . These variants covered 0.06 to $4.45 \%$ of the genome of each animal, with an average and median proportion of $1.6 \% \pm 0.7$ and $1.4 \%$, respectively (see Additional file 1: Table S1) and see Fig. 4. This observed variability across individuals can be partly explained by variations in coverage depth, which ranged from $8 \times$ to $33 \times$ (Fig. 5). Indeed, the smallest number of detected deletions and duplications was found for animals with a low sequencing depth and this increased as sequencing depth increased (Pearson correlation score $\rho=0.60, p$ value $<2.2 \mathrm{e}-16$ ).

Analysis of the distribution of variant size revealed that $75 \%$ of the deletion-only regions were shorter than $3.3 \mathrm{~kb}$ with a median size of $1.5 \mathrm{~kb}$ (Fig. 6), whereas $75 \%$ of the duplication-only regions were longer than $4.4 \mathrm{~kb}$, with a

Table 3 Number of predicted and validated variants

\begin{tabular}{lc}
\hline Type of variant & Number of variants \\
\hline Deletions & 3940 \\
Duplications & 67 \\
CNVR (deletion + duplication) & 171 \\
Total & 4178 \\
\hline
\end{tabular}

median size of $8.7 \mathrm{~kb}$. Likewise, about $75 \%$ of CNVR were longer than $45.7 \mathrm{~kb}$, with a median size of $114 \mathrm{~kb}$ (Fig. 6).

This finding confirms the results of a previous study in cattle [43] and may be explained by technical and/or biological factors. For example, our study only predicted tandem duplications because dispersed duplications are difficult to confirm by applying the combination of $\mathrm{CNV}$ detection tools that we used, thus the smaller number of detected duplications. In addition, some studies [44, 45] reported that certain recombination mechanisms, such as non-allelic homologous recombination (NHAR), result in more deletions than duplications, which may also partly explain the larger number of deletions observed in this work compared to that of duplications.

Further work is necessary to better analyze the link between different recombination mechanisms and the type of variants produced. Moreover, SR and PEM approaches were more sensitive for the detection of small variants because of the relatively small size of the insertions (321 bp on average). However, CNVnator, which uses an RD-based approach, is not limited by insert size, but in this study, we were constrained by the criterion that was set, i.e. that only variants predicted by at least two tools were retained. To balance this bias, future studies could use two RD tools to retain large-size variants. This technical bias could partly explain why the number of duplicated regions, which tend to be of larger size than deletions, was small compared to the number of deleted regions.

\section{Frequency of variants across animals and breeds}

The percentage of carriers for each variant varied from $0.5 \%$ (1 animal out of 200 ) to $97 \%$ (194 animals out of 200). Overall, $0.05 \%$ (two variants) were unique (observed in a single sample) (Fig. 7) and (see Additional file 9: Table S8), which suggests a very recent origin and confirms similar results that were reported in a study on Holstein [46]. The remaining $99.95 \%$ of detected variants (4176) were observed in at least $1 \%$ of the animals in our panel. These included variants that were shared among several animals within a single breed and variants that likely predated breed formation and were shared by two animal categories (beef and dairy).

Two variants located on BTA17 (CNVR_6167 and CNVR_6267) and a third one located on BTA6 (CNVR_15672) were observed in more than $90 \%$ of our population dataset. Analysis of the raw data for each tool revealed that the genome of the remaining $10 \%$ of the population also contained these CNV. However, they were discarded from the final results because they were detected with less than three reads, or predicted with only one tool, or did not pass the $70 \%$ reciprocal overlap filter. These CNV could also be specific to the 

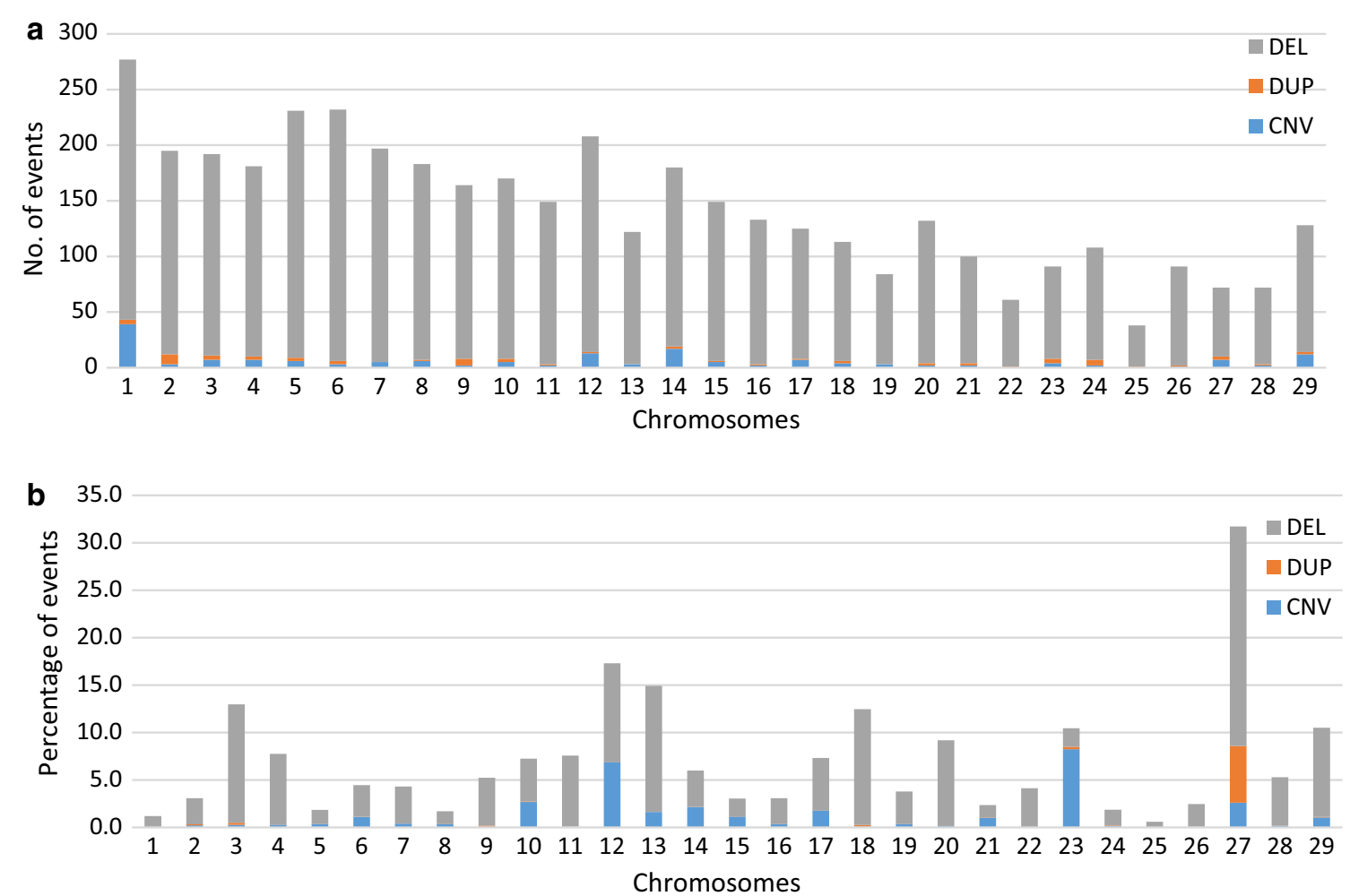

Fig. 3 Distribution of variants on autosomal chromosomes. a Number of variants per chromosome. b Proportion of chromosome covered by variants. Deletions are represented by grey, duplications by orange and CNV regions by blue bars

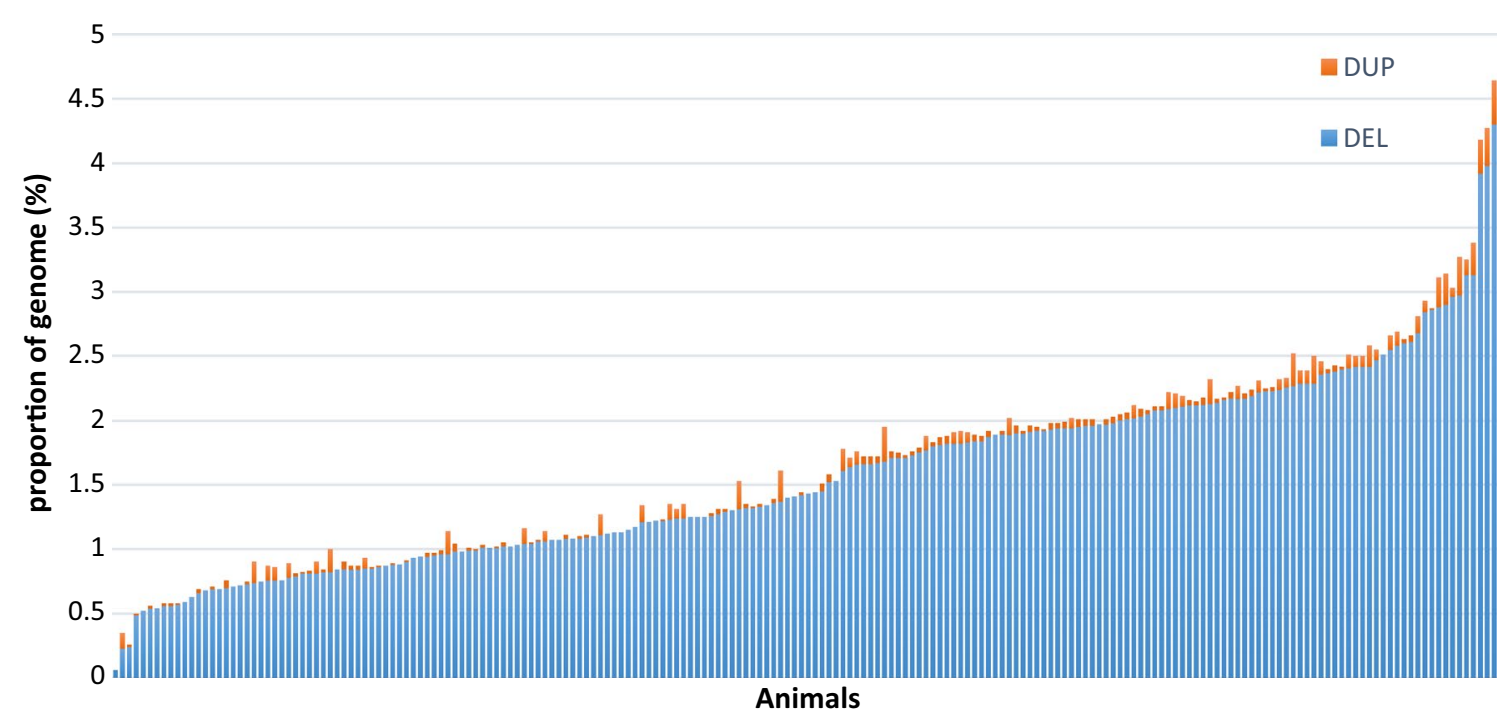

Fig. 4 Proportion of variants in each sample. Blue bars indicates the proportion of deletions and orange bars indicate the proportion of duplications in each sample 


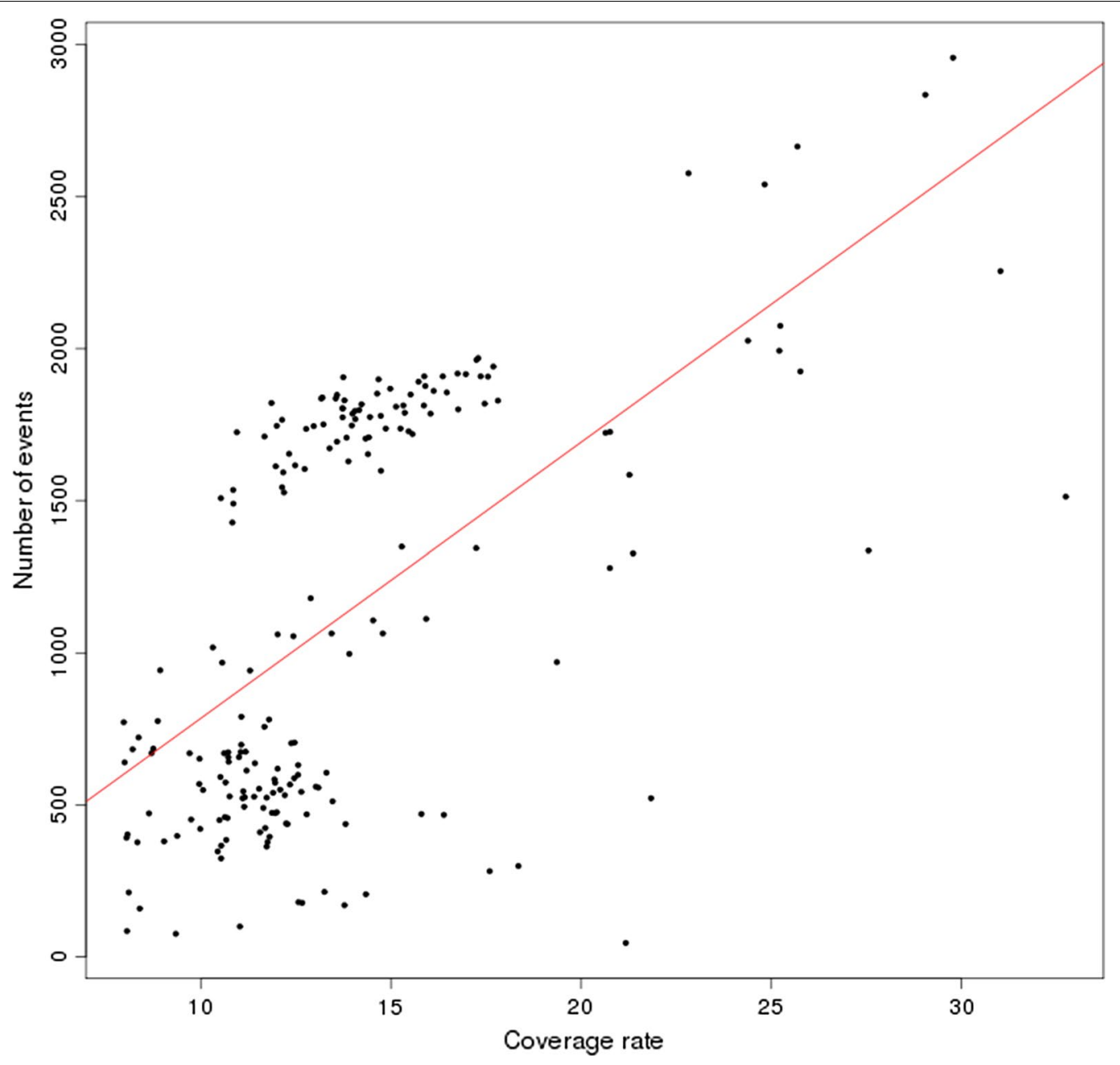

Fig. 5 Link between the number of variants and the coverage rate for each animal

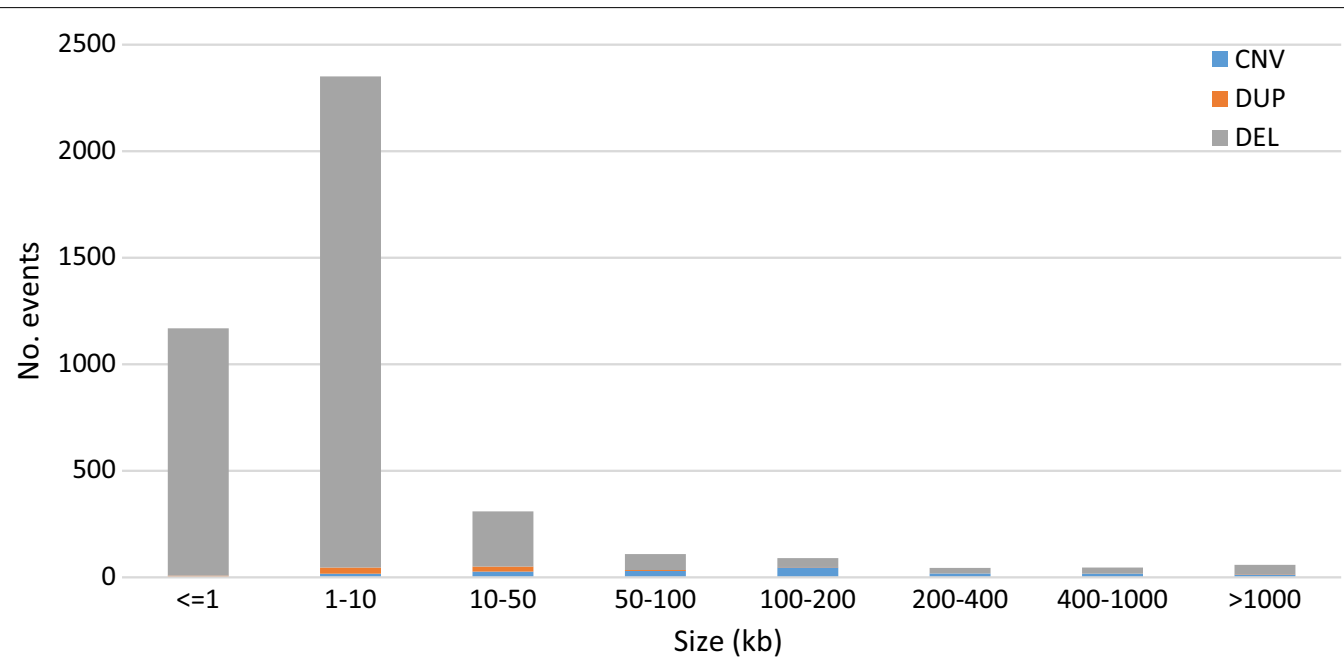

Fig. 6 Distribution of the size of the deletions, duplications and CNVR 


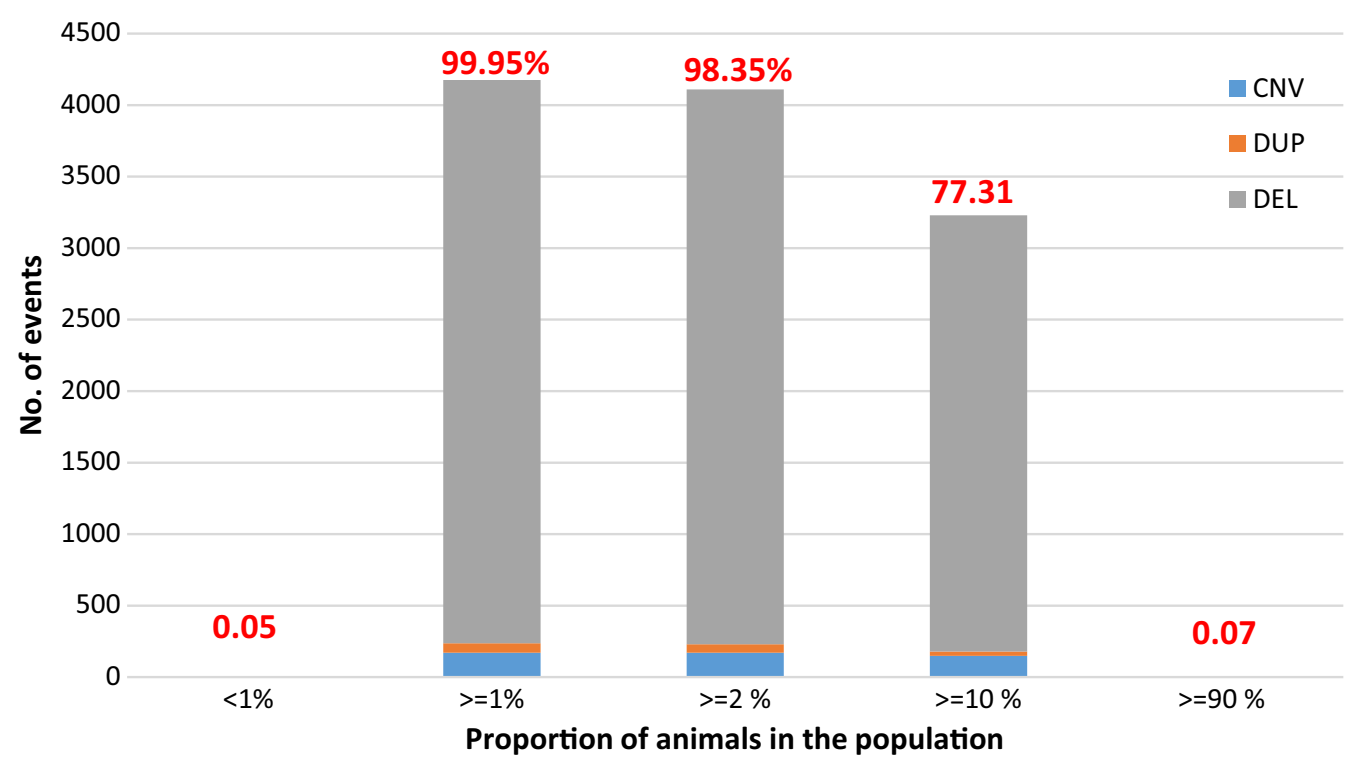

Fig. 7 Frequency of variants in the population $(N=200)$

individual that was used to produce the UMD3.1 reference sequence. Another explanation could be the presence of locally mis-assembled segments within the reference genome sequence. In addition, CNV prediction based on whole-genome sequences depends strongly on the quality of the bovine reference genome, which is known to be less good than the human reference genome.

The distribution of deletion, duplication, and CNV regions across breeds revealed that only $2.1 \%$ were breedspecific, $97.9 \%$ were observed in at least two breeds and $17.2 \%$ (717) were predicted and validated in the eight breeds analyzed (see Additional file 9: Table S8). Of these 717 variants, $92.5 \%$ (663) were deletion-only regions, $6.8 \%$ (49) were CNVR and $0.7 \%$ (5) were duplication-only regions. Since the UMD3.1 reference genome sequence was obtained from a Hereford animal, these deletiononly and duplication-only regions shared by all breeds could probably be Hereford- or even Dominette-specific events.

The distribution of variants across breeds was highly variable. Almost $96.12 \%$ of the variants (4016) were observed in both dairy and beef breeds, $0.17 \%$ (7) in only beef and $3.71 \%$ (155) in only dairy breeds (see Additional file 9: Table S8). The number of variants shared between breeds did not vary significantly $\left(\chi^{2}\right.$ test, $p$ value $=0.26$ ). We observed a small difference in the number of variants detected in dairy breeds only (155) and in beef breeds only (7), which can be partly explained by differences in the number of animals in each breed type and the coverage rate of sequencing used in the Mendelian and twice-sequenced approaches. The 16 pairs and two sire-son-dam trios used in the Mendelian approach were all dairy animals. In the twice-sequenced approach, we explored data from five dairy and nine beef animals, but the coverage rate was much higher for dairy (from $23 \times$ to $30 \times$ ) than for beef animals (from $9 \times$ to $13 \times$ ) (see Additional file 1: Table S1).

Most of the variants detected in beef breeds were shared by at least three breeds (3522 out of 4023, 86\%), while 2872 out of 4171 (69\%) were shared by a minimum of three dairy breeds (Fig. 8). The dairy breeds studied here have undergone strong selection to produce the best reproductive animals based on traits of interest; also, artificial insemination is frequently used in dairy breeds to disseminate the selected traits. Thus, each breed has a high degree of specialization, which may explain the small number of variants shared among dairy breeds (69\%). On the contrary, the distribution of variants within the beef breeds was very heterogeneous, probably because artificial insemination is much less used in beef breeds.

\section{Comparison of our dataset with previously described CNV}

We compared all deletion, duplication, and $\mathrm{CNV}$ regions identified in our study to publicly available data. Overall, 2278 regions $(54.5 \%)$ including 2102 deletion regions, 22 duplication regions, and 154 CNVR overlapped with publicly available results from seven published studies (Fig. 9).

Different factors related to the method used to identify CNV probably explain the relatively small proportion of common variants. Among these are the platforms 


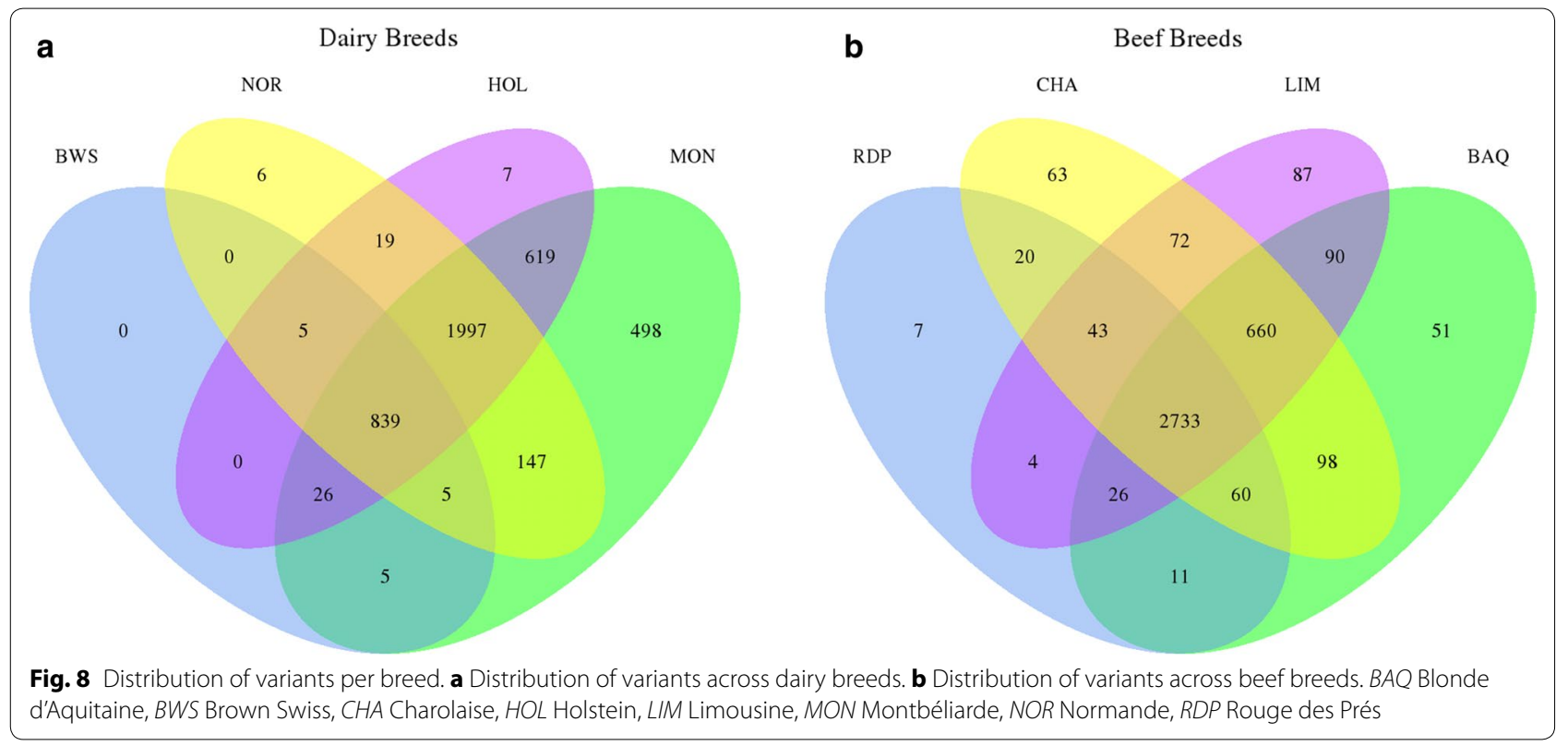

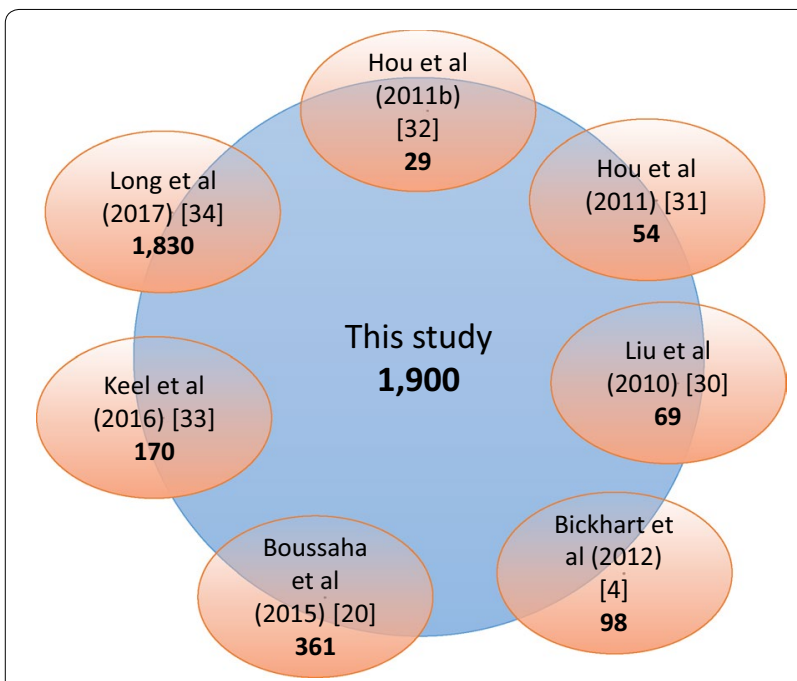

Fig. 9 Comparison of predicted variants with published studies. Our results were compared to studies derived from SNP arrays [31, 32], from CGH [30] and from whole-genome sequencing $[4,20,33,34]$. In this study we analyzed a large panel of breeds (four beef and four dairy breeds) compared to the other studies

and methods used for CNV calling, and/or the population size and structure of the studied animal populations, and the criteria used to define a $\mathrm{CNV}$ region. In a previous study, an array-based CGH approach was used to predict CNV in 90 animals from Bos taurus, Bos indicus and composite breeds [30] and found no small variants, i.e. the smallest identified CNV was 18,000 bp long. Whereas, in our study, the majority of variants were less than 18,000 bp long. In another study, Hou et al. reported $\mathrm{CNV}$ predicted from 472 Angus animals that were genotyped using a medium SNP genotyping array
(BovineSNP50) and they did not detect small variants (i.e. mean CNV size $=174,844$ bp) [32]. Since we did not include Angus cattle in our study, our results do not contain any Angus-specific CNV. Furthermore, 5.1 and $8.4 \%$ of the variants reported by Hou et al. were unique to either an individual or a breed, respectively. This suggests that many bovine $\mathrm{CNV}$ are yet to be discovered.

\section{Functional annotation of CNVR}

Analysis of the gene content of deletion-only, duplication-only, and CNV regions revealed that 1100 of all the variants (1000 deletion-only, 21 duplication-only, and 79 $\mathrm{CNV}$ regions) identified in our study contained or overlapped with 1803 genes (see Additional file 11: Table S10). Of these, $86 \%$ (1577 genes) corresponded to known protein-coding genes, 66 were pseudogenes, 87 genes were small nuclear and nucleolar RNA-coding genes, 26 were microRNA-coding genes, 38 were ribosomal RNAs, and nine were miscellaneous RNA coding genes. Around $81 \%$ (1460) of these genes had paralogs. In addition, 231 variants resulted in the deletion of an entire gene, nine in the duplication of an entire gene and 69 CNVR encompassed an entire gene. Furthermore, 186 of the deletion regions removed either partially or entirely a gene, which, in cattle, are described as lacking a paralog; thus, their deletion can alter gene expression and disturb the pathways in which they are involved. Further studies could target homozygous animals to study the effect of these deleted regions.

In order to identify the cellular functions associated with genes located in deletions, duplications, and CNVR, we performed a gene ontology (GO) analysis with 
PANTHER. The GO analysis classified 1442 genes into three GO categories: biological process, cellular component, and molecular function. These genes were enriched in a variety of cellular functions such as cellular and metabolic processes, binding, catalytic capacity, response to stimulus and cell part (Fig. 10). Because this set of genes is involved in a wide range of molecular functions, changes in gene copy number could result in a range of potential phenotypic variations among animals.

The genomic positions of the detected variants were also compared to the positions of publicly available QTL [36]. Overall, 1095 variants overlapped with QTL regions that are associated with milk (10 QTL), production (43 QTL), health (27 QTL), reproduction (26 QTL), or meat and carcass traits (73 QTL) (see Additional file 12: Table S11). In addition, 276 variants overlapped, partially or entirely, with both genes and QTL.
Several of the genes that were found to be located within the variants detected here are known to be associated with several important traits in cattle. One example is the Bardet-Biedl syndrome 7 (BBS7) gene, which is partially deleted by the CNVR_15659, has no paralog in the bovine genome and is co-localized with a known bovine QTL for body weight (see Additional file 12: Table S11). This gene is also associated with body weight and male infertility in mouse [47]. We identified this CNVR_15659 region in four dairy animals (one Holstein and three Montbéliarde) and nine beef animals (five Blonde d'Aquitaine, three Charolaise and one Limousine). Additional studies are needed to investigate the link between this deletion and the QTL for body weight.

A second CNVR_12945 region was found to entirely delete the HSD17B7 (hydroxysteroid 17-beta dehydrogenase 7) gene, which is related to heifer conception

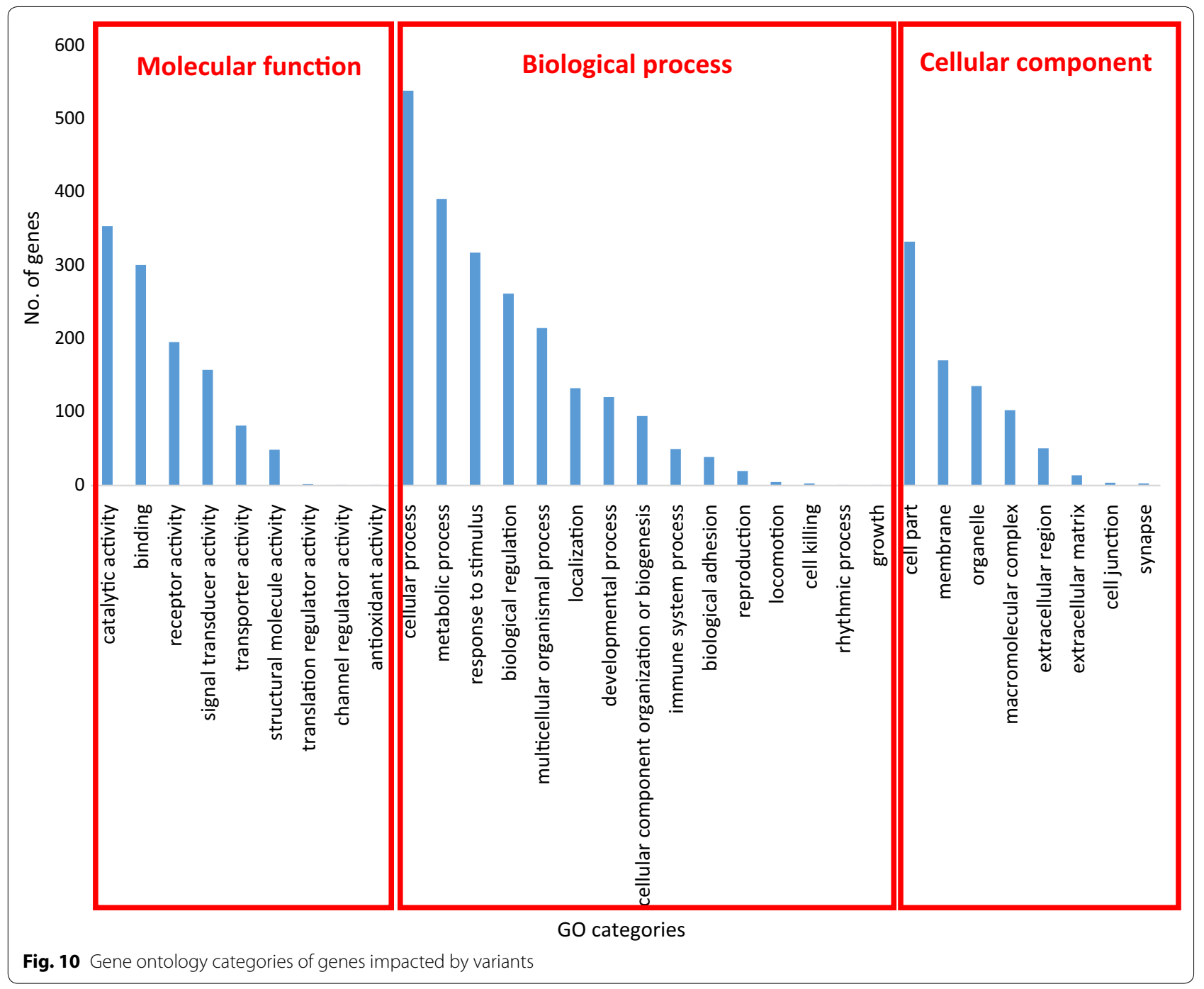


rate trait in Holstein cattle [48]. This region occurred at a higher frequency in beef than in dairy breeds; it was found in only four dairy animals (Montbéliarde) but in 32 beef animals (eight Blonde d'Aquitaine, 11 Charolaise, 10 Limousine and three Rouge des Prés). It would be very interesting to study the effect of this gene on heifer conception rate in the Montbéliarde breed.

Another interesting region, CNVR_10026, which encompasses entirely the SUPT3H (SPT3 homolog) and RUNX2 (runt related transcription factor) genes, occurred at different frequencies in beef and dairy breeds; it was deleted in 11 dairy animals (four Holstein, six Montbéliarde and one Normande) and 18 beef animals (seven Blonde d'Aquitaine, six Charolaise, four Limousine and one Rouge de Prés). Two more beef animals (one Blonde d'Aquitaine and one Rouge de Prés) had CNVR (both a duplication and deletion in the same region). SUPT3H and RUNX2 are associated with milk fat traits [49]. In addition, we found that $R U N X 2$ overlaps with a QTL associated with 305-day milk yield and milk protein percentage [50] and $S U P T 3 H$ is upstream of this same QTL.

The duplication CNVR_11771, which we detected in two Montbéliarde individuals, one Charolaise, one Limousine and one Rouge des Prés individual, overlapped with the transcription factor gene GTF2E2 (general transcription factor IIE subunit 2), which is known to be deregulated during Eimeria bovis infection [51].

In summary, we found that several of the variants detected in our study could potentially impact genes that are associated with important cattle traits. Future studies are needed to examine these variants in detail, together with phenotypic records, to confirm or infirm their effects.

\section{Conclusions}

Several recently developed NGS-based detection algorithms have led to significant progress in CNV detection. Here, we identified and characterized deletions, duplications, and CNV in eight French cattle breeds. This study represents one of the largest efforts for applying a sequence-based approach to detect $\mathrm{CNV}$ in cattle (200 animals). By exploring different complementary approaches and applying a stringent merge strategy, we identified 4178 deletion-only, duplicationonly, and CNV regions in both dairy and beef animals. We found 4163 variants by using two in silico approaches (Mendelian inheritance and reproducible predictions from sequences from multiple sequencing runs of the same animal). Of these 4178 variants, 69 were confirmed using SNP genotyping data and 15 other variants were validated using an array-based $\mathrm{CGH}$ approach. Our analyses revealed that predictions were most accurate when using a combination of Pindel + CNVnator tools. Some of the variants identified here can potentially affect genes that are involved in economically important cattle traits, and further analyses are necessary to investigate their possible effect. This study will contribute to drawing up a CNV map in French cattle and examining the potential impact of this kind of genetic variation on economically important traits of interest.

\section{Additional files}

Additional file 1: Table S1. Number of variants predicted for each individual. This table provides details on each sequenced animal: sequencing coverage, statistics about reads number, its parents if they were sequenced and the total number of predicted variants.

Additional file 2. $\mathrm{CGH}$ protocol. In this document, a detailed $\mathrm{CGH}$ protocol is provided.

Additional file 3: Table S2. Variants identified in the 200 whole-genome sequences. This file provides details on all predicted and merged variants before applying validation approaches (in silico and experimental).

Additional file 4: Table S3. Variants validated by the Mendelian approach. This table provides details on validated variants using the Mendelian approach and the number of trios, duos or parents with several sons that support the variant.

Additional file 5: Table S4. Percentage of validated variants in trios and parent-son pairs. This table includes statistics about the percentage of inherited variants from the parent to its son.

Additional file 6: Table S5. Variants validated by the twice-sequenced approach.

Additional file 7: Table S6. Variants identified by the array-based CGH approach. This table provides details on variants identified using the array-based CGH approach for 17 animals compared to whole-genome sequencing variants.

Additional file 8: Table S7. Selected variants for genotyping on the low-density Bovine BeadChip. In this table, we provide information for validated variants using the low-density Bovine BeadChip. Variants that were validated using the two in silico approaches and the array-based CGH are indicated.

Additional file 9: Table S8. Summary of validated variants by the different approaches. This is an inventory of validated variants using the two in silico and/or array-based CGH approaches. This dataset was the final subset of variants retained in this study. Information about breeds and validation approaches that support each variant is indicated.

Additional file 10: Table S9. Distribution of variants across chromosomes. This table provides variant counts and chromosome coverage for each bovine autosome.

Additional file 11: Table S10. Genes overlapping with identified deletion, duplication and CNV regions. In this table, we report genes that overlap entirely or partially with validated variants.

Additional file 12: Table S11. QTL overlapping with identified deletion, duplication and CNV regions. In this table, we report validated variants co-localized with QTL regions and details for each QTL.

\section{Authors' contributions}

$R L, D R$, and $M B$ conceived and designed the study. RL performed the bioinformatics and experimental analysis and drafted the manuscript. DR, MB, and $\mathrm{DB}$ contributed to the interpretation of the results and to the writing of the manuscript. DB, DR, SF, RP, and VB conceived the different whole-genome sequencing projects. CG, CM and ER extracted the DNA samples. DE, JB, and 
CK performed the whole-genome sequencing and constructed the sequence alignments. LT and RL performed the CGH experiments. All authors read and approved the final manuscript.

\section{Author details \\ 1 GABI, INRA, AgroParisTech, Université Paris-Saclay, 78352 Jouy-en-Josas, France. ${ }^{2}$ GMA, INRA, Université de Limoges, UMR1061, Unité Génétique Moléculaire Animale, 123 avenue Albert Thomas, 87060 Limoges Cedex, France. ${ }^{3}$ Allice, Maison Nationale des Eleveurs, 75012 Paris, France. ${ }^{4}$ LISBP, CNRS, INRA, INSA, Université de Toulouse, Toulouse, France. ${ }^{5}$ GenPhySE, INRA, Université de Toulouse INPT ENSAT, Université de Toulouse INPT ENVT, 52627 Castanet-Tolosan, France. ${ }^{6}$ SIGENAE, INRA, 52627 Castanet-Tolosan, France.}

\section{Acknowledgements}

This work was funded by INRA (GenSSeq and BovCNV projects from Selgen metaprogram, AAP-GA Mutated), the Agence Nationale de la Recherche (Regulomix ANR-09-GENM-011 and Cartoseq ANR-10-GENM-018), Apis-Gene (Cartoseq ANR-10-GENM-018, ReproSeq, SexSeq) and the Région Limousin (BovSeq). We wish to thank Tara G. McDaneld from the USDA, ARS, U.S. Meat Animal Research Center in the United States of America for the generous donation of Dominette reference DNA.

\section{Competing interests}

The authors declare that they have no competing interests.

\section{Publisher's Note}

Springer Nature remains neutral with regard to jurisdictional claims in published maps and institutional affiliations.

Received: 5 May 2017 Accepted: 17 October 2017

Published online: 24 October 2017

\section{References}

1. lafrate AJ, Feuk L, Rivera MN, Listewnik ML, Donahoe PK, Qi Y, et al. Detection of large-scale variation in the human genome. Nat Genet. 2004;36:949-51.

2. Sebat J, Lakshmi B, Troge J, Alexander J, Young J, Lundin P, et al. Largescale copy number polymorphism in the human genome. Science. 2004;305:525-8.

3. McCarroll SA, Altshuler DM. Copy-number variation and association studies of human disease. Nat Genet. 2007;39:S37-42.

4. Bickhart DM, Hou Y, Schroeder SG, Alkan C, Cardone MF, Matukumalli LK, et al. Copy number variation of individual cattle genomes using nextgeneration sequencing. Genome Res. 2012;22:778-90.

5. McCarroll SA, Huett A, Kuballa P, Chilewski SD, Landry A, Goyette P, et al. Deletion polymorphism upstream of IRGM associated with altered IRGM expression and Crohn's disease. Nat Genet. 2008;40:1107-12.

6. Wellcome Trust Case Control Consortium N, Craddock N, Hurles ME, Cardin N, Pearson RD, Plagnol V, et al. Genome-wide association study of CNVs in 16,000 cases of eight common diseases and 3,000 shared controls. Nature. 2010;464:713-20.

7. Gonzalez E, Kulkarni H, Bolivar H, Mangano A, Sanchez R, Catano G, et al. The influence of CCL3L1 gene-containing segmental duplications on HIV-1/AIDS susceptibility. Science. 2005;307:1434-40.

8. Johansson Moller M, Chaudhary R, Hellmén E, Höyheim B, Chowdhary B, Andersson L. Pigs with the dominant white coat color phenotype carry a duplication of the KIT gene encoding the mast/stem cell growth factor receptor. Mamm Genome. 1996;7:822-30.

9. Norris BJ, Whan VA. A gene duplication affecting expression of the ovine ASIP gene is responsible for white and black sheep. Genome Res. 2008;18:1282-93.

10. Wright D, Boije H, Meadows JRS, Bed'hom B, Gourichon D, Vieaud A, et al. Copy number variation in intron 1 of SOX5 causes the Pea-comb phenotype in chickens. PLoS Genet. 2009;5:e1000512.

11. Dorshorst B, Harun-Or-Rashid M, Bagherpoor AJ, Rubin CJ, Ashwell C, Gourichon D, et al. A genomic duplication is associated with ectopic eomesodermin expression in the embryonic chicken comb and two duplex-comb phenotypes. PLoS Genet. 2015;11:e1004947.

12. Salmon Hillbertz NHC, Isaksson M, Karlsson EK, Hellmén E, Pielberg GR, Savolainen P, et al. Duplication of FGF3, FGF4, FGF19 and ORAOV1 causes hair ridge and predisposition to dermoid sinus in Ridgeback dogs. Nat Genet. 2007;39:1318-20.

13. Drögemüller C, Distl O, Leeb T. Partial deletion of the bovine ED1 gene causes anhidrotic ectodermal dysplasia in cattle. Genome Res. 2001;11:1699-705.

14. Capitan A, Allais-Bonnet A, Pinton A, Marquant-Le Guienne B, Le Bourhis $\mathrm{D}$, Grohs $\mathrm{C}$, et al. A 3.7 Mb deletion encompassing ZEB2 causes a novel polled and multisystemic syndrome in the progeny of a somatic mosaic bull. PLOS ONE. 2012;7:e49084

15. Curtis C, Lynch AG, Dunning MJ, Spiteri I, Marioni JC, Hadfield J, et al. The pitfalls of platform comparison: DNA copy number array technologies assessed. BMC Genomics. 2009;10:588.

16. Valsesia A, Macé A, Jacquemont S, Beckmann JS, Kutalik Z. The growing importance of CNVs: new insights for detection and clinical interpretation. Front Genet. 2013;4:92

17. Alkan C, Coe BP, Eichler EE. Genome structural variation discovery and genotyping. Nat Rev Genet. 2011;12:363-76.

18. Pinto D, Darvishi K, Shi X, Rajan D, Rigler D, Fitzgerald T, et al. Comprehensive assessment of array-based platforms and calling algorithms for detection of copy number variants. Nat Biotechnol. 2011;29:512-20.

19. Boussaha M, Michot P, Letaief R, Hozé C, Fritz S, Grohs C, et al. Construction of a large collection of small genome variations in French dairy and beef breeds using whole-genome sequences. Genet Sel Evol. 2016;48:87.

20. Boussaha M, Esquerré D, Barbieri J, Djari A, Pinton A, Letaief R, et al. Genome-wide study of structural variants in bovine Holstein, Montbéliarde and Normande dairy breeds. PLoS ONE. 2015;10:e0135931.

21. Li H, Durbin R. Fast and accurate long-read alignment with BurrowsWheeler transform. Bioinformatics. 2010;26:589-95.

22. Abyzov A, Urban AE, Snyder M, Gerstein M. CNVnator: an approach to discover, genotype, and characterize typical and atypical CNVs from family and population genome sequencing. Genome Res. 2011;21:974-84.

23. Chen K, Wallis JW, McLellan MD, Larson DE, Kalicki JM, Pohl CS, et al. BreakDancer: an algorithm for high-resolution mapping of genomic structural variation. Nat Methods. 2009;6:677-81.

24. Ye K, Schulz MH, Long Q, Apweiler R, Ning Z. Pindel: a pattern growth approach to detect break points of large deletions and medium sized insertions from paired-end short reads. Bioinformatics. 2009;25:2865-71.

25. Rausch T, Zichner T, Schlattl A, Stütz AM, Benes V, Korbel JO. DELLY: structural variant discovery by integrated paired-end and split-read analysis. Bioinformatics. 2012;28:i333-9.

26. Baumbusch LO, Aarøe J, Johansen FE, Hicks J, Sun H, Bruhn L, et al. Comparison of the Agilent, ROMA/NimbleGen and Illumina platforms for classification of copy number alterations in human breast tumors. BMC Genomics. 2008;9:379.

27. Ghosh S, Qu Z, Das PJ, Fang E, Juras R, Cothran EG, et al. Copy number variation in the horse genome. PLoS Genet. 2014;10:e1004712.

28. Boichard D, Chung H, Dassonneville R, David X, Eggen A, Fritz S, et al. Design of a bovine low-density SNP array optimized for imputation. PLoS ONE. 2012:7:e34130.

29. Wang K, Li M, Hadley D, Liu R, Glessner J, Grant SFA, et al. PennCNV: an integrated hidden Markov model designed for high-resolution copy number variation detection in whole-genome SNP genotyping data. Genome Res. 2007:17:1665-74.

30. Liu GE, Hou Y, Zhu B, Cardone MF, Jiang L, Cellamare A, et al. Analysis of copy number variations among diverse cattle breeds. Genome Res. 2010:20:693-703.

31. Hou Y, Liu GE, Bickhart DM, Cardone MF, Wang K, Kim ES, et al. Genomic characteristics of cattle copy number variations. BMC Genomics. 2011;12:127.

32. Hou Y, Liu GE, Bickhart DM, Matukumalli LK, Li C, Song J, et al. Genomic regions showing copy number variations associate with resistance or susceptibility to gastrointestinal nematodes in Angus cattle. Funct Integr Genomics. 2012;12:81-92.

33. Keel BN, Keele JW, Snelling WM. Genome-wide copy number variation in the bovine genome detected using low coverage sequence of popular beef breeds. Anim Genet. 2017:48:141-50. 
34. Chen L, Chamberlain AJ, Reich CM, Daetwyler HD, Hayes BJ. Detection and validation of structural variations in bovine whole-genome sequence data. Genet Sel Evol. 2017;49:13

35. Quinlan AR, Hall IM. BEDTools: a flexible suite of utilities for comparing genomic features. Bioinformatics. 2010;26:841-2.

36. Hu ZL, Park CA, Reecy JM. Developmental progress and current status of the Animal QTLdb. Nucleic Acids Res. 2016:44:D827-33.

37. Thomas PD, Kejariwal A, Campbell MJ, Mi H, Diemer K, Guo N, et al. PANTHER: a browsable database of gene products organized by biological function, using curated protein family and subfamily classification. Nucleic Acids Res. 2003;31:334-41.

38. Hozé C, Fouilloux MN, Venot E, Guillaume F, Dassonneville R, Fritz S, et al. High-density marker imputation accuracy in sixteen French cattle breeds. Genet Sel Evol. 2013;45:33.

39. Boichard D, Maignel L, Verrier E. The value of using probabilities of gene origin to measure genetic variability in a population. Genet Sel Evol. 1997;29:5-23.

40. Boichard D. Pedig: a fortran package for pedigree analysis suited for large populations. In: Proceedings of the 7th World Congress on Genetics applied to Livestock Production: 19-23 August 2002; Montpellier. CDROM communication No. 28-13; 2002.

41. Wu Y, Tian L, Pirastu M, Stambolian D, Li H. MATCHCLIP: locate precise breakpoints for copy number variation using CIGAR string by matching soft clipped reads. Front Genet. 2013;4:157.

42. Layer RM, Chiang C, Quinlan AR, Hall IM. LUMPY: a probabilistic framework for structural variant discovery. Genome Biol. 2014;15:R84

43. Bae JS, Cheong HS, Kim LH, NamGung S, Park TJ, Chun J-Y, et al. Identification of copy number variations and common deletion polymorphisms in cattle. BMC Genomics. 2010;11:232.

44. Fadista J, Thomsen B, Holm LE, Bendixen C. Copy number variation in the bovine genome. BMC Genomics. 2010;11:284.
45. Turner DJ, Miretti M, Rajan D, Fiegler H, Carter NP, Blayney ML, et al. Germline rates of de novo meiotic deletions and duplications causing several genomic disorders. Nat Genet. 2008;40:90-5.

46. Liu GE, Van Tassel CP, Sonstegard TS, Li RW, Alexander LJ, Keele JW, et al. Detection of germline and somatic copy number variations in cattle. Dev Biol (Basel). 2008;132:231-7.

47. Zhang Q, Nishimura D, Vogel T, Shao J, Swiderski R, Yin T, et al. BBS7 is required for $B B S o m e$ formation and its absence in mice results in BardetBiedl syndrome phenotypes and selective abnormalities in membrane protein trafficking. J Cell Sci. 2013;126:2372-80.

48. Ortega MS, Denicol AC, Cole JB, Null DJ, Hansen PJ. Use of single nucleotide polymorphisms in candidate genes associated with daughter pregnancy rate for prediction of genetic merit for reproduction in Holstein cows. Anim Genet. 2016:47:288-97.

49. Buitenhuis B, Janss LL, Poulsen NA, Larsen LB, Larsen MK, Sørensen P. Genome-wide association and biological pathway analysis for milk-fat composition in Danish Holstein and Danish Jersey cattle. BMC Genomics. 2014;15:1112.

50. Yang Y, Li Q, Ju Z, Huang J, Zhou L, Li R, et al. Three novel single-nucleotide polymorphisms of complement component 4 gene (C4A) in Chinese Holstein cattle and their associations with milk performance traits and CH50. Vet Immunol Immunopathol. 2012;145:223-32.

51. Taubert A, Wimmers K, Ponsuksili S, Jimenez CA, Zahner H, Hermosilla C. Microarray-based transcriptional profiling of Eimeria bovis-infected bovine endothelial host cells. Vet Res. 2010:41:70.

\section{Submit your next manuscript to BioMed Central and we will help you at every step:}

- We accept pre-submission inquiries

- Our selector tool helps you to find the most relevant journal

- We provide round the clock customer support

- Convenient online submission

- Thorough peer review

- Inclusion in PubMed and all major indexing services

- Maximum visibility for your research

Submit your manuscript at www.biomedcentral com/submit
O Biomed Central 